\title{
CALIDAD DE VIDA RELACIONADA A LA COMPASIÓN, BURNOUT Y SOBRECARGA DEL CUIDADOR EN ENFERMERAS/OS BOLIVIANOS
}

\author{
COMPASSION-RELATED QUALITY OF LIFE, LABOR BURNOUT AND CAREGIVER \\ OVERLOAD IN BOLIVIAN NURSES
}

\author{
VANESKa Cindy APONTE DAZA ${ }^{1}$, Felipe E. GARCÍA ${ }^{2}$, Bismarck Pinto TAPIA ${ }^{3}$, \\ RUDDY ILLANES ${ }^{4}$, ERICK ROTH ${ }^{5}$ Y ROSSANA TABOADA ${ }^{6}$. \\ ${ }^{1}$ UNIVERSIDAD CATÓLICA BOLIVIANA, \\ ${ }^{2}$ UNIVERSIDAD SANTO TOMÁS, CHILE \\ ${ }^{3,4,5} 5{ }^{6}$ UNIVERSIDAD CATÓLICA BOLIVIANA
}

FECHA RECEPCIÓN: $11 / 05 / 2020$ FECHA ACEPTACIÓN: 1/10/2020

Para citar este artículo: Aponte, V., García, F., Pinto, B., Illanes, R., Roth, E., \& Taboada, R. (2020). Calidad de vida relacionada a la compasión, burnout y sobrecarga del cuidador en enfermeras/os bolivianos. Psychologia, 14(2), 49-59. https://doi.org/ $10.21500 / 19002386.4712$

\section{Resumen}

Las/os enfermeras/os desarrollan una actividad laboral centrada en el cuidado de los otros. Esta labor es fuente de satisfacción, pero también ha implicado niveles importantes de fatiga y deterioro en su calidad de vida. El propósito del presente estudio fue establecer los predictores de calidad de vida relacionada a la compasión en enfermeras/os de hospitales públicos de La Paz y El Alto, Bolivia. Para ello se consideraron como predictores la sobrecarga del cuidador, el burnout y características sociolaborales. Participaron 500 enfermeras/os de cuatro hospitales, de los cuales 82,4\% fueron mujeres, con un rango de edad de entre 21 y 61 años. Se utilizó un diseño no-experimental de tipo descriptivo y correlacional, y de corte transversal. Se encontró que el factor de burnout de agotamiento emocional, la sobrecarga del cuidador y el hecho de tener hijos predicen negativamente la calidad de vida relacionada a la compasión. Además, se observó una correlación negativa del agotamiento emocional con la edad y los años de experiencia laboral. Se discute sobre la necesidad de otorgar mejores condiciones laborales que alivien el desgaste, faciliten la interacción trabajo-familia y promuevan una mejor calidad de vida en enfermeras/os.

Palabras Clave: burnout, calidad de vida, cansancio, desgaste profesional enfermería, fatiga de compasión, test de Zarit

vaneska.cindy@gmail.com; cindyaponte86@gmail.com. 


\section{Abstract}

The nurses develop a work activity centered on the care of others. This work is not satisfying, but it has also involved important levels of fatigue and deterioration in the quality of life. The purpose of this study was to establish predictors of quality of life related to the compassion in nurses in public hospitals in La Paz and El Alto, Bolivia. For them, they considered themselves as predictors of caregiver burden, burnout, and socio-occupational characteristics. The 500 nurses from four hospitals participated, $82,4 \%$ of whom were women, with a range of ages between 21 and 61 years. A descriptive and correlational non-experimental design was used, and cross-sectional. The data was taken in a sole for what corresponds to a transversal study. If it is found that the burnout factor of emotional exhaustion, the overload of the caregiver and the length of having children negatively predicts the quality of life related to the compassion. Furthermore, a negative correlation was observed between age and years of work experience with emotional exhaustion. It discusses the need to improve the working conditions that alleviate wear and tear, facilitate the work-family interaction, and promote a better quality of life among nurses.

Keyword: Burnout, quality of life, fatigue, nursing professional burnout, compassion fatigue, Zarit test

\section{Introducción}

La calidad de vida de un trabajador es un constructo multidimensional que depende de condiciones tales como la salud, los ingresos, la red de apoyo social y la actividad física, entre otros factores (Martínez, Méndez, Secanilla, Benavente \& Sevilla, 2014). Hoy en día, la calidad de vida de los trabajadores de la salud ha sido tema de discusión debido a los efectos negativos en la salud mental del personal sanitario que se han observado en varios estudios relacionados con el enfrentamiento de pandemias y el riesgo en el que se involucran con estas tareas (Brooks et al., 2020), por lo que determinar los factores que inciden en una mejor calidad de vida resulta de suma relevancia.

Figley (2002) señala que la calidad de vida profesional de quien ejerce labores de cuidado de otros, que en adelante llamaremos calidad de vida relacionada a la compasión, es posible evaluarla a través de tres constructos interrelacionados: la satisfacción por compasión, la fatiga por compasión y el burnout por compasión. La satisfacción por compasión corresponde al placer derivado de ayudar a los demás. El burnout por compasión corresponde a los sentimientos de desesperanza y dificultades para trabajar de manera eficaz. La fatiga por compasión es un estado de tensión y preocupación por los traumas de los pacientes, por lo que constituye el costo que asume un cuidador como resultado del cuidado de los demás. La fatiga por compasión también ha sido descrita como el agotamiento físico y espiritual de presenciar e ir absorbiendo los problemas y el sufrimiento de los demás (Peery, 2010; Sabo, 2011; Hunsaker, 2015).

La fatiga por compasión fue identificada por primera vez por Joinson (1992) en un estudio de agotamiento en enfermeras que trabajaban en un departamento de emergencias, en quienes se observaron comportamientos como fatiga crónica, irritabilidad, temor al trabajo, dolencias físicas y falta de alegría en la vida.

La fatiga por compasión se relaciona con la exposición repetida al sufrimiento y la profunda implicación por parte del cuidador, por ejemplo, a través de la ayuda directa que debe brindar a pacientes con enfermedades terminales (Oliver, Sansó, Galiana, Tomás \& Benito, 2017) o que presentan un estrés postraumático primario (Yoder, 2010). Otros factores que incrementan y fomentan su aparición serían: a) el contacto continuo con la enfermedad, el dolor y la muerte; b) contacto continuo con usuarios que presentan problemas complejos; c) poca o nula formación para el manejo de aspectos emocionales de la persona que sufre y muere; d) dificultades de comunicación con pacientes (temas delicados, malas noticias); e) diferencias en valores y percepciones; f) dificultad de compartir el poder y de adherirse a un proyecto común; g) ralentización y falta 
de autonomía en la toma de decisiones, y h) exigencia de tiempo y de disponibilidad (Cáceres, 2018).

Por otro lado, el burnout por compasión corresponde al estado de agotamiento físico, emocional y mental ocasionado por la intervención a largo plazo en situaciones emocionalmente exigentes (Hunsaker, 2015; Wang, et al., 2020). El burnout por compasión también se acompaña de desilusión y sentimientos negativos; puede disminuir la confianza en la humanidad y frecuentemente conlleva altos niveles de vulnerabilidad; asimismo, afecta la calidad del tratamiento para los pacientes, disminuye la moral y aumenta el absentismo (Kelly, 2015).

Existe otra variable relacionada a la fatiga y el burnout por compasión, que es el burnout que las personas sienten por el ejercicio mismo de su trabajo, al cual, para distinguirlo del primer tipo de burnout descrito, se denominará en el presente estudio como burnout propiamente tal. De acuerdo con Maslach y Jackson (Citados por Calveche, 2013), el burnout se compone de tres dimensiones: agotamiento físico o psicológico, que se expresa en una vaga sensación de "no poder dar más de sí mismo" a quienes prestan servicio; b) despersonalización hacia los usuarios y compañeros de trabajo, mostrándose distanciado y con una considerable pérdida de motivación hacia su trabajo; c) baja realización personal, que implica la presencia de sentimientos de inadecuación personal y profesional al puesto de trabajo, que surge de la comprobación de que no son capaces de atender debidamente las demandas de su tarea.

En un estudio realizado en enfermeras españolas (Martín, 2015) se observó una alta prevalencia en las profesionales de todas las dimensiones del burnout laboral, por lo que es un malestar que aqueja a un número importante de enfermeras/os.

El agotamiento manifestado en el burnout y la fatiga por compasión propia del cuidador, si bien están estrechamente relacionados, difieren en algunos aspectos centrales. Las definiciones de agotamiento con mayor frecuencia apuntan a estresores ambientales, mientras que las definiciones de fatiga por compasión abordan la naturaleza relacional de la condición. Además, el agotamiento se asocia con sentimientos de desesperanza y apatía y crea una incapacidad para desempeñar sus tareas de manera efectiva (Mashego, 2016; Maila, Martin \& Chipps, 2020); en cambio, la fatiga por compasión está más vinculada a la empatía (Hidalgo, Martínez \& Carrasco, 2018). El agotamiento y la fatiga por compasión pueden convertirse en cargas abrumadoras en enfermeras/ os, y causar problemas físicos, mentales y emocionales, al igual que dificultades en la salud (Hunsaker, 2015).

Tanto la fatiga por compasión como el agotamiento puede causar que una enfermera/o se vuelva ineficaz, deprimida, apática y desapegada (Boyle, 2011). Resultados a largo plazo en agotamiento incluyen baja moral en el lugar de trabajo, ausentismo y apatía, presentando serios desafíos en todos los niveles de la atención médica (Jones, 2007; Portnoy, 2011). Todo esto tiene un impacto negativo tanto en el profesional afectado como en la atención al paciente (Portnoy, 2011).

Los cuidadores profesionales deben aprender a reconocer los signos de agotamiento y fatiga por compasión, debido al riesgo de padecerlo que se ha reportado en los estudios ya citados. Si los síntomas de agotamiento y fatiga por compasión, por ejemplo, resfriados frecuentes, dolores de cabeza y mal humor (Rodríguez, 2016), no son tratados oportunamente, pueden derivar en quejas somáticas, despersonalización, cinismo, irritabilidad y baja energía (Plieger et al., 2015). Con el tiempo, los cuidadores pueden sentirse desilusionados, abrumados y volverse fríos en su atención, llegando a experimentar crisis de salud, o presencia de sintomatología ansiosa o postraumática (Moss, 2016).

En las/os enfermeras/os, la alta carga de trabajo, horarios inadecuados, afrontamiento de situaciones emocionalmente intensas asociadas con la enfermedad y el sufrimiento de pacientes y familiares, entre otras, requiere de habilidades de empatía y de autocompasión, pues si no logran equilibrar adecuadamente sus habilidades con las características propias de su trabajo, es más probable que las/os afecte el agotamiento y la fatiga por compasión (Bambula \& Gómez, 2016). La autocompasión, por ejemplo, ha demostrado ser un factor protector para una amplia gama de indicadores de bienestar y se ha asociado con la compasión por los demás (Auserón et al., 2018).

La calidad de vida relacionada a la compasión también puede verse influida por los niveles de sobrecarga de trabajo, definida como la percepción de un empleado de que le han dado más trabajo del que se puede completar dentro de un período de tiempo determinado, siendo 
uno de los factores más importantes que contribuyen al estrés laboral (Othman \& Nasurdin, 2013; Shirom \& Melamed, 2006).

La sobrecarga de trabajo suele ser el resultado de un aumento en el número de pacientes a cargo, así como la falta de recursos económicos y reducciones de personal (Rauhala et al., 2006; Vegsund, 2014). Además, las/os enfermeras/os también están expuestas, a diario, a interacciones emocionalmente exigentes, tales como confrontación con pacientes y sus familias, y exposición a muerte, enfermedad y sufrimiento (Bakker \& Heuven, 2006). Aunque la investigación previa se ha centrado principalmente en los efectos negativos de las demandas emocionales sobre el estrés laboral, estudios recientes han proporcionado evidencia de un efecto positivo sobre el compromiso, especialmente cuando los recursos para realizar el trabajo están disponibles (De Jonge et al., 2008; Gabel-Shemueli, Dolan \& Ceretti, 2017).

Ante estos antecedentes, el objetivo del presente estudio fue evaluar la influencia de la sobrecarga del cuidador, el burnout y características socio-laborales sobre la calidad de vida relacionada a la compasión en enfermeras/ os de hospitales públicos de La Paz y El Alto, Bolivia.

\section{Método}

Diseño.

Se utilizó un diseño no-experimental de tipo descriptivo y correlacional. Los datos fueron tomados de una sola vez, por lo que corresponde a un estudio transversal.

Participantes.

Participaron 500 enfermeras/os que llevaban entre 1 a 25 años en la atención de pacientes y que cumplen entre 21 a 45 horas de trabajo a la semana. Del total, un $82,4 \%$ fueron mujeres y un $17,6 \%$ hombres, con un rango de edad de entre 21 y 61 años $(M=36,72$; $D E=8,91)$.

Instrumentos.

Sobrecarga del cuidador. Se utilizó el test de Zarit (Lanz et al., citado por Breinbauer et al., 2009), el cual consta de 22 ítems que abarcan distintas dimensiones relacionadas a la carga del cuidador, como son sus redes de apoyo, calidad de vida, capacidad de autocuidado y competencias para el cuidado (Schulz \& Beach, 1999; Crespo \& Rivas, 2015). Se contesta en una escala Likert que va desde 0 (nunca) a 4 (casi siempre), según la frecuencia con la que el participante se perciba en la situación descrita. De acuerdo con Barreto-Osorio et al. (2015), puntajes inferiores a 46 puntos indican que no existe sobrecarga, y una superior a 56 es indicador de sobrecarga. En el estudio realizado por Albarracín (2016) con cuidadores colombianos se obtuvo una fiabilidad de $\alpha=0.88$ para la escala total. En otro estudio con cuidadores argentinos, se estableció una adecuada validez de constructo a través de un análisis factorial confirmatorio (Tartaglini et al., 2020).

Calidad de vida relacionada a la compasión. Se utilizó la escala Pro-QOL v-IV-Versión Traducida- (Stamm, 2005, citado por Martínez-González, 2014), un instrumento con 30 ítems que evalúa la calidad de vida profesional a través de tres subescalas: satisfacción por compasión, burnout por compasión y fatiga por compasión. Para el cálculo de la escala total se invirtieron los puntajes de burnout por compasión y fatiga por compasión. Se contesta en una escala Likert de 6 puntos, desde 0 (nunca) hasta 5 (muy a menudo). En el estudio realizado por Cuartero (2019) en España, la escala mostró una consistencia interna adecuada, de $\alpha=0,87$ para satisfacción, de $\alpha=0,80$ para fatiga y de $\alpha=0,72$ para burnout.

Burnout laboral. Se evaluó a través del Inventario de Burnout de Maslach, versión traducida (Maslach \& Jackson, 1986, citado por Roth, 2010), el cual consta de 22 ítems y está compuesto de tres subescalas: agotamiento emocional, despersonalización y bajo logro personal. Se responde en una escala Likert de siete puntos que varía de 0 (nunca experimenté tal sentimiento) a 6 (experimenté tales sentimientos todos los días). En un estudio en Bolivia se obtuvo una consistencia interna satisfactoria para la subescala de agotamiento emocional, con un $\alpha=0,84$, y débil para la subescala de despersonalización y bajo logro personal, con un $\alpha=0,65$ y 0,67, respectivamente (Roth, 2010).

\section{Procedimiento.}

El estudio fue de tipo censal, es decir, se buscó encuestar a todas las enfermeras que trabajaban dentro de cuatro hospitales públicos de La Paz y El Alto, en Bolivia, lo que corresponde a la población objetivo. Este 
método permite contar con un indicador real de los niveles de burnout, sobrecarga y calidad de vida en estos profesionales. Para ello, se contó con la colaboración de cada uno de los hospitales, lo que permitió acceder a 500 enfermeras/os, de un total de 648 registradas, lo que equivale al $77 \%$ del total.

Previo a la aplicación de los cuestionarios, las/os enfermeras/os firmaron un consentimiento informado, donde se explicaba la naturaleza del estudio y se les aseguraba la confidencialidad y el anonimato. Luego de esto, se les entregó el set de instrumentos a la jefa de enfermeras/os, quien repartió las pruebas y cada persona contestó de manera privada y en el momento que tenían libre. El tiempo medio utilizado en la aplicación fue de 30 minutos.

En este estudio fueron considerados los principios éticos habituales la investigación psicológica de acuerdo con la American Psychological Association (2010).

Análisis de datos.

En primer lugar, se realizó un análisis descriptivo de las variables de estudio. Luego de eso, se buscó establecer la relación entre las variables de estudio utilizando la correlación $r$ de Pearson, la $t$ de Student y la prueba ANOVA. Para establecer los predictores de calidad de vida relacionada a la compasión, se utilizó una Regresión Lineal Múltiple, de tipo jerárquica. Se utilizó para ello el paquete estadístico IBM SPSS Statistic, versión 25.

\section{Resultados}

En la Tabla 1 se pueden observar los estadísticos descriptivos de cada escala utilizada en el estudio. En ella se observa que los niveles de consistencia interna de las escalas y subescalas es adecuada, con excepción de burnout-despersonalización y calidad de vida-burnout por compasión que son más bajos que el límite de lo aceptable. Los estadísticos de asimetría y curtosis muestran que todas las variables, a excepción de calidad de vida-satisfacción por compasión, mostraron niveles \pm 2, valores recomendados por Bollen y Long (1994) para el uso de estadísticos paramétricos. Al aplicar la prueba de Kruskal-Wallis, se confirma la distribución no paramétrica de calidad de vida-satisfacción por compasión, por lo que en los análisis de correlaciones y regresión se utilizaron sus puntajes z.

\section{Tabla 1}

Estadísticos descriptivos y consistencia interna de las variables $(n=500)$.

\begin{tabular}{lccccccc}
\hline \multicolumn{1}{c}{ Variable } & Mín. & Máx. & M & DE & Asimetría & Curtosis & $\boldsymbol{\alpha}$ \\
\hline 1. Burnout & 9 & 105 & 61,84 & 16,21 & $-0,05$ & 0,80 & 0,74 \\
a. Despersonalización & 0 & 32 & 11,57 & 7,27 & 0,71 & $-0,41$ & 0,45 \\
b. Agotamiento & 2 & 46 & 20,16 & 8,52 & 0,57 & $-0,05$ & 0,71 \\
c. Baja realización & 2 & 42 & 31,89 & 8,00 & $-1,29$ & 1,69 & 0,75 \\
2. Calidad de vida & 53 & 105 & 77,81 & 10,27 & 0,11 & $-0,57$ & 0,73 \\
a. Satisfacción por compasión & 12 & 40 & 33,23 & 4,92 & $-1,66$ & 4,46 & 0,82 \\
b. Burnout por compasión & 11 & 39 & 26,35 & 4,16 & $-0,27$ & 1,00 & 0,46 \\
c. Fatiga por compasión & 6 & 36 & 18,23 & 6,20 & 0,14 & $-0,75$ & 0,76 \\
3. Sobrecarga & 3 & 69 & 29,89 & 12,84 & 0,87 & 1,20 & 0,88 \\
\hline \hline
\end{tabular}

Al analizar las relaciones entre las variables (ver Tabla 2) se encontró que la escala de burnout correlacionó en forma inversa con la escala de calidad de vida $(r=-0,28)$ y directa con sobrecarga $(r=0,23)$. A su vez, la calidad de vida presenta una correlación inversa con sobrecarga $(r=-0,26)$. Llama la atención la correlación más bien baja, aunque significativa, entre burnout y burnout por compasión $(\mathrm{r}=0,24)$, lo que podría indicar que se trata de constructos relacionados pero diferentes. 
Tabla 2

Correlaciones $r$ de Pearson entre las variables de estudio $(n=500)$

\begin{tabular}{|c|c|c|c|c|c|c|c|c|}
\hline Variable & $1 \mathbf{a}$ & $1 b$ & $1 \mathrm{c}$ & 2 & $2 \mathbf{a}$ & $2 b$ & $2 \mathrm{c}$ & 3 \\
\hline 1. Burnout & $* * * 0,66$ & $* * * 0,78$ & $* * * 0,62$ & $* * *-0,28$ & 0,01 & $* * * 0,24$ & $* * * 0,26$ & $* * * 0,23$ \\
\hline a. Despersonalización & na & $* * * 0,52$ & 0,06 & $* * *-0,21$ & $-0,03$ & $* * * 0,21$ & $* * * 0,20$ & $* * * 0,18$ \\
\hline b. Agotamiento & & $\mathrm{Na}$ & $* 0,09$ & $* * *-0,30$ & $-0,06$ & $* * * 0,32$ & $* * * 0,31$ & $* * * 0,35$ \\
\hline c. Baja realización & & & na & $-0,07$ & $* 0,09$ & $-0,02$ & 0,03 & $-0,04$ \\
\hline 2. Calidad de vida & & & & na & $* * * 0,43$ & $* * *-0,56$ & $* * *-0,88$ & $* * *-0,26$ \\
\hline a. Satisfacción por compasión & & & & & na & $* * *-0,27$ & $-0,08$ & 0,01 \\
\hline b. Burnout por compasión & & & & & & na & $* * * 0,60$ & $* * * 0,34$ \\
\hline c. Fatiga por compasión & & & & & & & na & $* * * 0,25$ \\
\hline 3. Sobrecarga & & & & & & & & na \\
\hline
\end{tabular}

$* \mathrm{p}<0,05 ; * * * \mathrm{p}<0,001 ;$ na $=$ no aplica

Considerando las variables sociodemográficas y laborales, se encontró una correlación significativa del agotamiento emocional con la edad $(\mathrm{r}=-0,12 ; \mathrm{p}=$ $0,007)$ y con la antigüedad laboral $(r=-0,11 ; p=0,13)$. Los años de experiencia también presenta una correlación positiva con agotamiento emocional $(\mathrm{r}=-0,12 ; \mathrm{p}=$ $0,01)$. A través de la $t$ de Student también se encontró que las/os enfermeras/os que tenían hijos presentaban más fatiga por compasión y menos calidad de vida relacionada a la compasión que quienes no tenían hijos.

Finalmente, se realizó una Regresión Lineal Múltiple de tipo jerárquica, en dos pasos, para establecer en qué medida las variables incluidas en este estudio predecían una mejor calidad de vida relacionada a la compasión. En este análisis se incluyeron, en un primer paso, las variables sociodemográficas de edad, antigüedad laboral y presencia de hijos, de forma de controlar su influencia sobre la calidad de vida relacionada a la compasión. En un segundo paso, se incluyeron las variables psicológicas de sobrecarga del cuidador y burnout laboral. El modelo obtenido en el segundo paso fue significativo, obteniendo un $\mathrm{R}^{2}=0,14, \mathrm{~F}$ $(7,492)=11,007, \mathrm{p}<0,001$. En este modelo resultaron predictores negativos significativos de la calidad de vida el tener hijos $(\beta=-0,11)$, la sobrecarga del cuidador $(\beta=-0,18)$ y el agotamiento emocional $(\beta=-0,19)$.

Tabla 3

$R L M$ jerárquica en calidad de vida relacionada a la compasión $(n=500)$

\begin{tabular}{|c|c|c|c|c|c|}
\hline & \multicolumn{2}{|c|}{ Coeficientes no estandarizados } & \multirow{2}{*}{$\begin{array}{c}\text { Coeficientes tipificados } \\
\beta\end{array}$} & \multirow{2}{*}{ Valor $\mathrm{t}$} & \multirow{2}{*}{ Valor $\mathbf{p}$} \\
\hline & B & Error típico & & & \\
\hline \multicolumn{6}{|l|}{ Paso $1 . R^{2}=0,02, p=0,03$} \\
\hline (Constante) & $-78,74$ & 2,32 & & $-33,872$ & $<0,001$ \\
\hline Edad & 0,08 & 0,08 & 0,07 & 0,931 & 0,352 \\
\hline Antigüedad laboral & 0,04 & 0,09 & 0,03 & 0,383 & 0,702 \\
\hline Tener hijos $\left(\mathrm{No}_{0}=0 ;\right.$ Sí $\left.=1\right)$ & $-3,13$ & 1,11 & $-0,14$ & $-2,820$ & 0,005 \\
\hline \multicolumn{6}{|c|}{ Paso 2. $\mathrm{R}^{2}=0,14 ; \Delta \mathrm{R}^{2}=0,12 ; \mathrm{p}<0,001$} \\
\hline (Constante) & $-66,05$ & 3,19 & & $-20,687$ & $<0,001$ \\
\hline Edad & 0,03 & 0,08 & 0,03 & 0,435 & 0,664 \\
\hline Antigüedad laboral & 0,02 & 0,09 & 0,01 & 0,218 & 0,828 \\
\hline Tener hijos $($ No $=0$; Sí = 1) & $-2,45$ & 1,05 & $-0,11$ & $-2,334$ & 0,020 \\
\hline Sobrecarga & $-0,15$ & 0,04 & $-0,18$ & $-4,050$ & $<0,001$ \\
\hline
\end{tabular}




\begin{tabular}{lcccccc}
\hline & \multicolumn{2}{c}{ Coeficientes no estandarizados } & Coeficientes tipificados & \multirow{2}{*}{ Valor t } & \multirow{2}{*}{ Valor $\mathbf{p}$} \\
\cline { 2 - 6 } & $\mathbf{B}$ & Error típico & $\boldsymbol{\beta}$ & $-0,07$ & $-1,404$ & 0,161 \\
Burnout - Despersonalización & $-0,13$ & 0,09 & $\mathbf{- 0 , 1 9}$ & $\mathbf{- 3 , 6 5 4}$ & $<\mathbf{0 , 0 0 1}$ \\
Burnout - Agotamiento emocional & $\mathbf{- 0 , 2 1}$ & $\mathbf{0 , 0 6}$ & $-0,06$ & $-1,315$ & 0,189 \\
Burnout - Baja realización personal & $-0,06$ & 0,05 & & \\
\hline \hline
\end{tabular}

Nota: En negrilla los predictores significativos.

\section{Discusión}

Este estudio tenía como propósito establecer los predictores de calidad de vida relacionada a la compasión en enfermeras/os de hospitales públicos de la ciudad de La Paz en Bolivia. La calidad de vida relacionada a la compasión de las enfermeras/os, así como la sobrecarga del cuidador y el burnout resultan de vital importancia, considerando la cantidad de exigencias laborales que reporta el trabajo específico de la enfermería, cuyo sentido dice relación con el cuidado de los otros.

La enfermería es una profesión particularmente estresante, lo que afecta tanto la salud y el bienestar personal como la satisfacción laboral y colectiva. Esto tiene aún mayor relevancia considerando que los graves problemas de salud que han azotado el mundo, y en particular a Bolivia en estos últimos tiempos, con el COVID-19 entre ellos, hacen a las/losenfermeras/os una población altamente susceptible para presentar problemas de salud mental, incluso más que los contagiados por la enfermedad o quienes deben hacer cuarentena obligatoria (Brooks et al., 2020).

Los resultados de este estudio indican la presencia de tres predictores principales de la calidad de vida relacionada a la compasión de las/os profesionales: tener hijos, la sobrecarga del cuidador y el agotamiento emocional como factor del burnout.

Se observó que las/os enfermeras/os que tenían hijos presentaban una menor calidad de vida relacionada a la compasión que quienes no tenían hijos, coincidiendo con lo señalado en otros estudios (Quintana et al., 2016). Estos resultados se explicarían, ya que las funciones dentro de la casa constituyen una carga adicional al trabajo remunerado; el cuidado de los hijos puede quitar tiempo al descanso o al ocio, impidiendo recuperarse tras largas horas de trabajo. Marín et al. (2004) observaron que estar casada no influía en una mejor calidad de vida y que muchas veces el único apoyo son las personas que trabajan en las mismas labores que ellas, personas que están igualmente afectadas.

Por otro lado, la sobrecarga del cuidador se relaciona a una menor calidad de vida relacionada a la compasión. Este resultado coincide con lo encontrado por Andrade (2019), donde explica que el exceso de actividades del cuidador influye en un deterioro de su calidad de vida. Una posible explicación es que los turnos de trabajo, el tener que trabajar en varios lugares y el poco tiempo libre afectan al rendimiento laboral, más aún si deben lidiar además con enfermedades que amenazan la vida, en donde en muchas ocasiones debe actuar de intermediarios entre el médico y la familia de los pacientes. Las diferentes rotaciones dentro del hospital, el tener que estar atenta al cuidado de cada paciente y el deber de no fallar en la adecuada atención, genera mucha sobrecarga. A esto se añade la ambigüedad de roles, por no existir especificidad de funciones y tareas, la falta de autonomía y autoridad para la toma de decisiones, los cambios tecnológicos, las malas relaciones interpersonales y la superposición familia-trabajo, los que contribuyen a aumentar las tensiones específicas del quehacer hospitalario, produciendo mayor sobrecarga en los/as enfermeras/os (Rendón \& Vargas, 2019).

A su vez, el agotamiento emocional predijo una peor calidad de vida relacionada a la compasión, tal como también se encontró en Puello et al. (2014). Es posible que esto se deba al escaso acceso a actividades de entretenimiento, la falta de autonomía sobre la planificación de horarios (incluyendo turnos rotativos) y una falta de conformidad con el salario percibido, dada la carga laboral. Estos signos de fatiga pueden afectar la relación con las personas que los rodean y el manejo de sus emociones tanto dentro como fuera del trabajo.

Otro hallazgo interesante, ya más a nivel teórico, es la baja correlación entre el burnout por compasión y el 
burnout propio del desgaste profesional. Esto se debería a que, si bien están relacionados, se trata de constructos separados. El primero se relaciona con el agotamiento físico, mental y emocional que genera el cuidar a un grupo de personas por varias horas (Soto, Barrios \& Molina, 2017); en cambio, el segundo se relaciona con el estrés laboral crónico integrado por actitudes y sentimientos negativos hacia las personas con las que se trabaja y hacia el propio rol profesional, así como la vivencia de encontrarse emocionalmente agotado (Arias \& González, 2009; Blanca \& Arias, 2018). Dicha diferencia justifica la inclusión de los dos indicadores de burnout en el presente estudio.

También resulta interesante la relación inversa existente entre la edad y los años de experiencia, con el agotamiento emocional. Los hallazgos muestran que esta relación no está establecida, pues mientras algunos autores coinciden con este resultado (Nespereira \& Vázquez, 2017; Bilbao, et al., 2018; Camargo \& Hernández, 2020), otros han encontrado la relación contraria, es decir, más edad y años de experiencia, más agotamiento emocional o burnout (Abreu, 2011; García, Maldonado \& Ramírez, 2014; Velazco, 2019). En el actual estudio, este resultado podría deberse a que la experiencia laboral permite ir construyendo estrategias de afrontamiento más efectivas para lidiar con la sobrecarga laboral y con el consiguiente agotamiento, desde la aceptación de aquello que no se puede cambiar, hasta estrategias más activas para disminuir el cansancio en el cumplimiento de sus funciones. Las estrategias específicas que usan los/as enfermera/os de mayor edad y antigüedad versus las de menos edad y experiencia, es un interesante tema que podría abordarse en un estudio posterior con las mismas participantes.

Este estudio tiene algunas limitaciones que es necesario señalar. En primer lugar, la baja fiabilidad de dos subescalas impide extraer resultados concluyentes en los análisis que incluyen dichas escalas. Una segunda limitación es su naturaleza transversal, pues impide establecer relaciones de causa y efecto; por ello, este estudio muestra más bien la influencia relativa de una variable sobre otra.

Se recomienda que en futuros estudios se evalúen condiciones sociodemográficas que no fueron incluidas en el presente trabajo, como los horarios, el tiempo de permanencia en las unidades, el sistema de turnos, además de otras variables psicológicas, como la satisfacción laboral, las estrategias de afrontamiento al estrés y la interacción trabajo-familia, entre otras. Por último, dado que este estudio tiene la ventaja de haber encuestado a un gran número de enfermeras/ os, equivalente al $77 \%$ del total de profesionales de cuatro importantes hospitales de La Paz y El Alto, abre la posibilidad de volver a encuestarlos para evaluar los cambios producidos por la crisis del coronavirus.

En conclusión, este estudio aporta en establecer los factores que influyen en una disminución de la calidad de vida relacionada a la compasión en enfermeras/os de la ciudad de la Paz y el Alto en Bolivia. La influencia negativa de la existencia de hijos es un llamado de atención a la forma en que se organiza su trabajo, que impide compatibilizar adecuadamente las labores domésticas con las exigencias del trabajo formal y que debe ser abordado por las instituciones hospitalarias. A su vez, la sobrecarga generada por el cuidado y el agotamiento emocional implican que la carga laboral no está debidamente regulada. Se requiere un sistema de turnos que respete los límites físicos y mentales de los/as enfermeras/os, dado que cumplen una función de vital importancia para la salud de los pacientes y sus familiares, por lo que el cuidado de su propia salud es esencial para ejercer sus tareas con sentido y satisfacción.

\section{Referencias}

Abreu, F. (2011). Síndrome de agotamiento emocional en profesionales de la unidad de cuidados intensivos. Multiciencias, 11 (4), 370-377.

Albarracín, A. C. (2016). Escala de sobrecarga del cudiador Zarit: estructura factorial en cuidadores informales de Bucaramanga. Revista de Psicologia (Universidad de Antioquía), 8(2), 87-99. https://doi. org/ 10.17533/udea.rpsua.v8n2a06

American Psychological Association (2010). Ethical principles of psychologists and code of conduct. Washington DC, USA: APA.

Andrade, C.. (2019). Cuidadores de pacientes demenciales y su estado de salud mental. (Tesis de titulación). Universidad Técnica de Machala, Ecuador.

Arias, G. F., \& Gonzáles, M. (2009). Estrés, agotamiento profesional (burnout) y salud en profesores de acuerdo con su tipo de contrato. Ciencia y Trabajo, $11(31), 172-176$. 
Arrogante, O., \& Aparicio-Zaldivar, E. G. (2020). Síndrome de burnout en los profesionales de cuidados intensivos: relaciones con la salud y el bienestar. Enfermería Intensiva, 3 1(2), 60-70. https://doi. org/ 10.1016/j.enfi.2019.03.004

Auserón, G. A., Viscarret, M. R. E., Goñi, C. F., Rubio, V. G., \& Pascual, P. P. (2018). Evaluación de la efectividad de un programa de mindfulness y autocompasión para reducir el estrés y prevenir el burnout en profesionales sanitarios de atención primaria. Atención Primaria, 50(3), 141-150. https://doi.org/ 10.1016/j.aprim.2017.03.009

Bakker, A.B., \& Heuven, E. (2006). Emotional dissonance, burnout, and in-role performance among nurses and police officers. International Journal of Stress Management, 13(4), 423-440. https://doi. org/ 10.1037/1072-5245.13.4.423

Barreto-Osorio, R., Campos de Aldana, M., Carrillo, G., Coral, R., Chaparro, L., Durán, M., Rosales, R., \& Ortiz, V. (2015). Entrevista Percepción de Carga del Cuidado de Zarit: pruebas psicométricas para Colombia. Aquichan, 15(3), 368-380. https://doi. org/ 10.5294/aqui.2015.15.3.5.

Bambula, F. D., \& Gómez, I. C. (2016). La investigación sobre el síndrome de burnout en Latinoamérica entre 2000 y el 2010. Psicología desde el Caribe, 33(1), 113-131. https://doi.org/ 10.14482/ psdc.33.1.8065

Bilbao, M., Martínez-Zelaya, G., Pavez, J., \& Morales, K. (2018). Burnout en trabajadores de ONGs que ejecuta política social en Chile. Psicoperspectivas, 17(3), 199-210. https://doi.org/ 10.5027/psicoperspectivas/vol17-issue3-fulltext-1454

Blanca, J., \& Arias. A. (2018). Sindrome de burnout en personal de enfermería: Asociación con estresores del entorno hospitalario, Andalucía, España. Enfermería Universitaria, 15(1), 30-44. https://doi.org/ 10.22201/eneo.23958421e.2018.1.62903

Breinbauer, H., Vásquez, H., Mayanz, S., Guerra, C., \& Millán, T. (2009). Validación en Chile de la Escala de Sobrecarga del Cuidador de Zarit en sus versiones original y abreviada. Revista Médica de Chile, 137(5), 657-665 https://doi.org/ 10.4067/ s0034-98872009000500009
Bollen, K., \& Long, J. S. (1994). Testing structural equation models. Newbury Park, CA: Sage.

Boyle, D. A. (2011). Countering compassion fatigue: A requisite nursing agenda. Online Journal of Issues in Nursing, 16(1), 1-16. https://doi.org/ 10.3912/ OJIN.Vol16No01Man02

Brooks, S. K., Webster, R. K., Smith, L. E., Woodland, L., Wessely, S., Greenberg, N., \& Rubin, G. J. (2020). The psychological impact of quarantine and how to reduce it: Rapid review of the evidence. The Lancet, 395(10.227), 912-920. https://doi.org/ 10.1016/S0140-6736(20)30460-8

Cáceres, J. M. (2018). Programa de prevención de desgaste por empatía en profesionales sanitarios de una unidad de diálisis de hospital. (Tesis de maestría). Universidad Autónoma de Madrid, España.

Calveche, S., Chazatar, L., Jiménez, E., \& Quiñones, R. (2013). Factores de riesgo asociados al Síndrome de Burnout en estudiantes de Odontología de la Universidad del Valle. Revista Estomatología y Salud, 21(1), 7-11. https://doi.org/ 10.25100/ re.v21i1.5752

Camargo, K., \& Hernández, J. (2020). Sindrome de Burnout en enfermería geríatrica: Revision de las perspectivas teóricas y prácticas. Revista Ciencia y Cuidado, 17(2), 1-9. https://doi.org/ 10.22463/17949831.1578

Crespo, M., \& Rivas, M. T. (2015). La evaluación de la carga del cuidador: Una revisión más allá de la escala de Zarit. Clínica y Salud, 1, 9-15. https://doi. org/ 10.1016/j.clysa.2014.07.002

Cuartero, M. E. (2019). Estudio sobre la prevalencia del desgaste por empatía (compassion fatigue) en los/as trabajadores/as sociales de los Centros de Servicios Sociales de Mallorca. (Tesis doctoral). Universitat de les Illes Balears, España.

De Jonge, J., Le Blanc, P.M., Peeters, M.C., \& Noordam, H. (2008). Emotional job demands and the role of matching job resources: A cross-sectional survey study among health care workers. International Journal of Nursing Studies, 45(10), 1460-1469. https://doi.org/ 10.1016/j.ijnurstu.2007.11.002

Figley, C. R. (2002). Treating compassion fatigue. American Journal of Clinical Hypnosis, 47(2), 131-133. https:// doi.org/ 10.1080/00029157.2004.10403632 
Gabel-Shemueli, R., Dolan, S., \& Ceretti, A. S (2017). Work conditions and engagement among nurses in Uruguay. Academia Revista Latinoamericana de Administración, 30(1), 59-71. https://doi.org/ 10.1108/ ARLA-02-2016-0049

García, B., Maldonado, S., \& Ramírez, M. (2014). Estados afectivos emocionales (depresión, ansiedad y estres) en personal de enfermería del sector salud pública de México. Summa Psicológica UST, 11 (1), 66-73.

Hidalgo, P., Martínez, S., \& Carrasco, M. (2018, octubre). Reduciendo la fatiga por compasión en cuidadores formales a través de una intervención cognitiva-existencial. Ponencia presentada en el III Congreso: Ciencia, Sociedad e Investigación Universitaria, Pontificia Universidad Católica del Ecuador, Ambato.

Hunsaker, S. C. (2015). Factors that influence the development of compassion fatigue, Burnout, and compassion satisfaction in emergency department nurses. Journal of Nursing Scholarship, 47(2), 186194. https://doi.org/ 10.1111/jnu.12122

Joinson, C. (1992). Coping with compassion fatigue. Nursing, 22(1), 16-120.

Jones, C., \&. (2007). The costs and benefits of nurse turnover: A business case for nurse retention. Online Journal of Issues in Nursing, 12(3). https://doi. org/ 10.3912/OJIN.Vol12No03Man04

Kelly, L. R. (2015). Predictors of compassion fatigue and compassion satisfaction in acute care nurses. Journal of Nursing Scholarship, 47(6), 522-528. https:// doi.org/ 10.1111/jnu.12162

Maila, S., Martin, P. D., \& Chipps, J. (2020). Professional quality of life amongst nurses in psychiatric observation units. South African Journal of Psychiatry, 26(1), 1-7. https://doi.org/ 10.4102/sajpsychiatry.v26i0.1553

Marín, M. J. A., Hernández, M. E. R., Moreno, M. D. G., Pérez, E. C., Gámez, A. G., Martínez, I. G., \& Piña, S. A. (2004). Apoyo social, características sociodemográficas y burnout en enfermeras y auxiliares de hospital. Enfermería Clínica, 14(5), 281-285. https://doi.org/10.1016/S11308621(04)73899-1

Martín, R. (2015). Burnout en enfermería: Prevalencia y factores relacionados en el medio hospitalario.
Revista Científica de la Sociedad Española de Enfermería Neurológica, 41, 9-14. https://doi.org/ 10.1016/j. sedene.2015.02.001

Martínez-González, A. S. (2014). Fatiga por Compasión. Escalas, medidas y mecanismos de prevención. (Tesis de grado). Universitat de les Illes Balears, España.

Martínez, J. P., Méndez, I., Secanilla, E., Benavente, A., \& Sevilla, J. G. (2014). Burnout en cuidadores profesionales y calidad de vida en residentes de centros institucionalizados. European Journal of Investigation in Health, Psychology and Education, 4(1), 41-53. https://doi.org/ 10.30552/ejihpe.v4i1.60

Mashego, T. A. (2016). Burnout, compassion fatigue and compassion satisfaction among nurses in the context of maternal and perinatal deaths. Journal of Psychology in Africa, 26(5), 469-472. https://doi. org/ 10.1080/14330237.2016.1219566

Moss, M. G. (2016). A critical care societies collaborative statement: Burnout syndrome in critical care health-care professionals. A call for action. American Journal of Respiratory and Critical Care Medicine, 194(1), 106-113. https://doi.org/ 10.4037/ ajcc2016133

Nespereira, T., \& Vázquez, M. (2017). Inteligencia emocional y manejo del estrés en profesionales de enfermería del servicio de urgencia hospitalaria. Enfermería Clínica, 27(3), 172-178. https://doi.org/ 10.1016/j.enfcli.2017.02.007

Oliver, A., Sansó, N., Galiana, L., Tomás, J. M., \& Benito, E. (2017). Evaluación psicométrica en profesionales de enfermería de cuidados paliativos. Aquichan, 17(2), 183-194. https://doi.org/ 10.5294/ aqui.2017.17.2.7.

Othman, N., \& Nasurdin, A.M. (2013). Social support and work engagement: A study of Malaysian nurses. Journal of Nursing Management, 21 (8), 1083-1090. https://doi.org/ 10.1111/j.13652834.2012.01448.x

Peery, A. I. (2010). Caring and burnout in registered nurses: What is the Connection? International Journal for Human 14(2), 53-60. https://doi.org/ 10.20467/1091-5710.14.2.52

Plieger, T., Melchers, M., Montag, C., Meermann, R., \& Reuter, M. (2015). Life stress as potential risk factor for depression and burnout. Burnout Re- 
search, 2(1), 19-24. https://doi.org/ 10.1016/j. burn.2015.03.001

Portnoy, D. (2011). Burnout and compassion fatigue: Watch for the signs. Health Progress, 92(4), 46-50.

Puello, Y., Quintero, N., Cánova, C., Camargo, Y., Amaya, L., Guzmán, Y., \& Cervantes, S. (2014). Calidad de vida percibida por el personal de enfermería de las unidades de cuidados intensivos de una clínica privada de la ciudad de Santa Marta. Investigación de Enfermería: Imagen y Desarrollo, 16(1), 87-99. https://doi.org/ 10.11144/Javeriana.IE16-1.cvpp

Quintana, M. A., Carrijo, K., Figueroa C., García, J., Salazar, R., Tinajero, R., Padilla M., \& Valle, C. (2016). Calidad de vida laboral de enfermeras de un hospital privado. Biotecnia, 18(1), 34-38. https://doi.org/ 10.18633/bt.v18i1.226

Rauhala, A., Kivimäki, M., Fagerström, L., Elovainio, M., Virtanen, M., Vahtera, J., Rainio, A.K., Ojaniemi, K. \& Kinnunen, J. (2006). What degree of work overload is likely to cause increased sickness absenteeism among nurses? Evidence from the RAFAELA patient classification system. Journal of Advanced Nursing, 57(3), 286-295. https://doi.org/ 10.1111/j.1365-2648.2006.04118.x

Rendón, C. \& Vargas, M. (2019). El precio de la vocación en el personal de enfermería y su familia. Revista Cubana de Enfermería, 35(2). Recuperado de http:// www.revenfermeria.sld.cu/index.php/enf/article/ view/1998

Rodríguez, L. M. (2016). Burnout syndrome in family caregivers of dependents. International Journal of Asian Social Science, 6(1), 12-20. https://doi.org/ 10.18488/journal.1/2016.6.1/1.1.12.20

Roth, E. P. (2010). Síndrome de burnout, personalidad y satisfacción laboral en enfermeras de la ciudad de La Paz. Ajayu, 8(2), 62-100.

Sabo, B. (2011). Reflecting on the concept of compassion fatigue. Online Journal of Issues in Nursing, 16(1), 1-22. https://doi.org/10.3912/OJIN.Vol16No01Man01

Shirom, A., \& Melamed, S. (2006). A comparison of the construct validity of two burnout measures in two groups of professionals. International Journal of Stress Management, 13(2), 176-200. https://doi. org/ 10.1037/1072-5245.13.2.176
Schulz, R., \& Beach, S. (1999). Caregiving as a risk factor for mortality. The caregiver health effects study. JAMA, 281(23), 2215-2219. https://doi. org/10.1001/jama.282.23.2215

Soto, P., Barrios, S. \& Molina, Y. (2017). Síndrome de quemarse por el trabajo y satisfacción laboral como predictores de calidad de la atención de enfermería hospitalaria. Ciencia y Enfermeria, 23(3), 99-111. https://doi.org/ 10.4067/S071795532017000300099

Tartaglini, M. F., Feldberg, C., Hermida, P. D., Heisecke, S. L., Dillon, C., Ofman, S. D., ... \& Somale, V. (2020) (2020). Escala de sobrecarga del cuidador de Zarit: análisis de sus propiedades psicométricas en cuidadores familiares residentes en Buenos Aires, Argentina. Neurologia Argentina, 12(1), 27-35. https://doi.org/ 10.1016/j.neuarg.2019.11.003

Vegsund, H. (2014). Work engagement among nurses. (Master's thesis). Norwegian University of Science and Technology, Norway.

Velazco, K. (2019). Factores epidemiológicos y laborales asociados al síndrome de agotamiento laboral en enfermeras de los servicios de emergencia y cuidados intensivos del hospital III Yanahuara es salud Arequipa-2014. Revista Médica Hosptital Hipólito Unanue, 12(2), 28-32.

Wang, J., Okoli, C. T., He, H., Feng, F., Li, J., Zhuang, L., \& Lin, M. (2020). Factors associated with compassion satisfaction, burnout, and secondary traumatic stress among Chinese nurses in tertiary hospitals: A cross-sectional study. International Journal of Nursing Studies, 102, 103472. https://doi. org/ 10.1016/j.ijnurstu.2019.103472

Yoder, E. (2010). Compassion fatigue in nurses. Applied Nursing Research, 24(4), 191-197. https://doi.org/ 10.1016/j.apnr.2008.09.003 